\title{
On the Eigenvalues of General Sum-Connectivity Laplacian Matrix
}

\author{
Hanyuan Deng · He Huang · Jie Zhang
}

Received: 9 May 2013 / Revised: 2 August 2013 / Accepted: 7 August 2013 /

Published online: 6 September 2013

(C) Operations Research Society of China, Periodicals Agency of Shanghai University, and

Springer-Verlag Berlin Heidelberg 2013

\begin{abstract}
The connectivity index was introduced by Randić (J. Am. Chem. Soc. 97(23):6609-6615, 1975) and was generalized by Bollobás and Erdös (Ars Comb. 50:225-233, 1998). It studies the branching property of graphs, and has been applied to studying network structures. In this paper we focus on the general sum-connectivity index which is a variant of the connectivity index. We characterize the tight upper and lower bounds of the largest eigenvalue of the general sum-connectivity matrix, as well as its spectral diameter. We show the corresponding extremal graphs. In addition, we show that the general sum-connectivity index is determined by the eigenvalues of the general sum-connectivity Laplacian matrix.
\end{abstract}

Keywords Connectivity index $\cdot$ Eigenvalue $\cdot$ Laplacian matrix

This work was supported by the Danish National Research Foundation and the National Science Foundation of China (No. 61061130540) for the Sino-Danish Center for the Theory of Interactive Computation and by the Center for Research in Foundations of Electronic Markets (CFEM, supported by the Danish Strategic Research Council), within which this work was performed.

H. Deng $\cdot$ H. Huang

College of Mathematics and Computer Science, Key Laboratory of High Performance Computing and Stochastic Information Processing (HPCSIP) (Ministry of Education of China), Hunan Normal University, Changsha, Hunan 410081, China

H. Deng

e-mail: hydeng@hunnu.edu.cn

H. Huang

e-mail: hehuang099@gmail.com

J. Zhang ( $\square)$

Computer Science Department, Aarhus University, Aarhus, Denmark

e-mail: jiezhang@cs.au.dk 


\section{Introduction}

Topological indices are numerical parameters of a graph which characterize the topological structure of the graph and are usually graph invariants. A nice property of these topological indices is that most of them can be calculated in polynomial time, including the connectivity index, the Wiener index, and Balaban's $J$ index, etc.

In general, the transformation of graphs into topological indices facilitates the manipulation of information and the search for structure-function relationships in studying complex networks. For example, González-Díaz et al. [8] reviewed and commented on the challenges and new trends in the applications of connectivity index in networks.

Connectivity index, as one of the most well-known topological indices, was introduced by Randić [12] in 1975 and was generalized by Bollobás and Erdös [2]. It studies the branching property of graphs. Bollobás and Erdös [2] proved that the connectivity index of a graph of order $n$ without isolated vertices is at least $\sqrt{n-1}$; they left the open question of the minimum value of the connectivity index for graphs $G$ with given minimum degree $\delta(G)$. Delorme et al. [4] answered this question for $\delta(G)=2$, thus partially solved the question. Furthermore, they proved a best possible lower bound on the connectivity index of a triangle-free graph $G$ with given minimum degree $\delta(G)$. Balister et al. [1] built up a technique to determine the maximal connectivity index of a tree with a specified number of vertices and leaves. Rodríguez [15] found the relationship between the high order connectivity index and its Laplacian spectrum, and bounded the high order connectivity index by the eigenvalues of the adjacency matrix and the Laplacian matrix of $G$. Reviews of mathematical properties of the connectivity index refer to $[9,10]$.

Although the mathematical properties of connectivity index have been intensively studied, its application in computer science is just at the very start but begins to attract much more attention.

The intrinsical properties of connectivity index coincide with the fundamental issues in studying ad-hoc networks, such as connectivity, scalability, routing and topology control. In particular, connectivity index has been used in studying connectivity properties of ad-hoc networks [11]. Ghimire et al. [7] estimated how well each node connects to the rest of the network by employing connectivity index. They also made use of connectivity index to select the supernodes that create a P2P overlay over ad-hoc networks. Their simplicial modeling led to a high robustness in the overlay network and decreased the overlay disconnection probability.

Riera-Fernández et al. [14] studied the assessment of the connectivity quality in new complex networks by making use of topological indices such as connectivity index and Wiener index. Estrada et al. [5] studied resistance distance, information centrality, node vulnerability and vibrations in complex networks by employing the connectivity index. Ranjan et al. [13] measured the sensitivity of connectivity index with respect to local perturbations in the network structures.

In this paper we focus on the general sum-connectivity matrix and the induced general sum-connectivity index, which is a variant of connectivity index. We show the tight upper and lower bounds of the largest eigenvalue of the general sumconnectivity matrix, as well as the tight upper bound of its spectral diameter. We 
further build up the relationship between the general sum-connectivity index and the general sum-connectivity Laplacian spectrum. We show that the general sumconnectivity index can be bounded by the eigenvalues of the Laplacian matrix.

\section{Preliminary}

Let $G$ be simple undirected graph with vertex set $V(G)=\left\{v_{1}, \cdots, v_{n}\right\}$ and edge set $E(G)=\left\{e_{1}, \cdots, e_{m}\right\}$. Let us denote $v_{i} v_{j} \in E$ if $v_{i}$ and $v_{j}$ are adjacent in graph $G$. Let $d_{i}$ be the degree of vertex $v_{i}, i=1, \cdots, n$. Let us denote $\mathrm{A}(G)=\left[a_{i j}\right]_{n \times n}$ the adjacency matrix of $G$. Unless otherwise specified we consider non-empty connected graph throughout the paper. We use $\mathbf{e}=(1, \cdots, 1)$ to denote the all-1 vector and use $\mathrm{J}$ to denote the all-1 matrix.

The celebrated product-connectivity index of graph $G$ was introduced by Randic in 1975 [12].

Definition 2.1 Given any graph $G$, the product-connectivity index of $G$ is

$$
\mathcal{X}(G)=\sum_{v_{i} v_{j} \in E} \frac{1}{\sqrt{d_{i} d_{j}}},
$$

where the sum is over all edges $v_{i} v_{j}$ of the graph $G$.

The product-connectivity matrix is $\mathrm{R}(G)=\left[r_{i j}\right]_{n \times n}$, where

$$
r_{i j}= \begin{cases}\frac{1}{\sqrt{d_{i} d_{j}}}, & \text { if } v_{i} v_{j} \in E \\ 0, & \text { otherwise }\end{cases}
$$

It is easy to see that $\mathcal{X}(G)=\frac{1}{2} \sum_{i=1}^{n} \sum_{j=1}^{n} r_{i j}$. Bollobás and Erdös [2] introduced the general product-connectivity index. It is denoted by

$$
\mathcal{X}_{\alpha}(G)=\sum_{v_{i} v_{j} \in E}\left(d_{i} d_{j}\right)^{\alpha},
$$

where $\alpha$ can be any real number. In addition, the original product-connectivity index is the special case that $\alpha=-\frac{1}{2}$. The general product-connectivity matrix is $\mathrm{R}_{\alpha}(G)=$ $\left[r_{i j}^{\alpha}\right]_{n \times n}$, where

$$
r_{i j}^{\alpha}= \begin{cases}\left(d_{i} d_{j}\right)^{\alpha}, & \text { if } v_{i} v_{j} \in E \\ 0, & \text { otherwise }\end{cases}
$$

Obviously, $\mathcal{X}_{\alpha}(G)=\frac{1}{2} \sum_{i=1}^{n} \sum_{j=1}^{n} r_{i j}^{\alpha}$.

Parallel to product-connectivity index, Zhou and Trinajstić $[16,17]$ introduced the sum-connectivity index. 
Definition 2.2 Given any graph $G$, the sum-connectivity index of $G$ is

$$
\mathcal{Z}(G)=\sum_{v_{i} v_{j} \in E} \frac{1}{\sqrt{d_{i}+d_{j}}},
$$

where the sum is over all edges $v_{i} v_{j}$ of $G$.

The sum-connectivity matrix is $\mathrm{S}(G)=\left[s_{i j}\right]_{n \times n}$, where

$$
s_{i j}= \begin{cases}\frac{1}{\sqrt{d_{i}+d_{j}}}, & \text { if } v_{i} v_{j} \in E ; \\ 0, & \text { otherwise }\end{cases}
$$

We thereby generalize the sum-connectivity index as follows.

Definition 2.3 Given any graph $G$, the general sum-connectivity index of $G$ is

$$
\mathcal{Z}_{\alpha}(G)=\sum_{v_{i} v_{j} \in E}\left(d_{i}+d_{j}\right)^{\alpha},
$$

where the sum is over all edges $v_{i} v_{j}$ of $G$.

The general sum-connectivity matrix is $\mathrm{S}_{\alpha}(G)=\left[s_{i j}^{\alpha}\right]_{n \times n}$, where

$$
s_{i j}^{\alpha}= \begin{cases}\left(d_{i}+d_{j}\right)^{\alpha}, & \text { if } v_{i} v_{j} \in E \\ 0, & \text { otherwise }\end{cases}
$$

In this paper we will focus on the general sum-connectivity index and eigenvalues of general sum-connectivity matrix. We study their properties and relationships. Note that the eigenvalues of the general sum-connectivity matrix $\mathrm{S}_{\alpha}(G)$ are real numbers since that $\mathrm{S}_{\alpha}(G)$ is real symmetric matrix. Denote the eigenvalues of $\mathrm{S}_{\alpha}(G)$ by $\mu_{1}, \cdots, \mu_{n}$. W.l.o.g., let us assume $\mu_{1} \leqslant \cdots \leqslant \mu_{n}$. The following lemma will be useful throughout the paper. The trivial proof of the lemma is omitted.

Lemma 2.1 The eigenvalues $\mu_{1}, \cdots, \mu_{n}$ of the general sum-connectivity matrix $\mathrm{S}_{\alpha}(G)$ satisfy

$$
\begin{aligned}
& \sum_{i=1}^{n} \mu_{i}=\operatorname{tr}\left(\mathrm{S}_{\alpha}(G)\right)=0 \\
& \sum_{i=1}^{n} \mu_{i}^{2}=\sum_{i=1}^{n} \sum_{j=1}^{n}\left(\mathrm{~s}_{i j}^{\alpha}\right)^{2}=2 \sum_{v_{i} v_{j} \in E}\left(d_{i}+d_{j}\right)^{2 \alpha}
\end{aligned}
$$

\section{Tight Bounds of the Eigenvalues of the General Sum-Connectivity Matrix}

In this section, we discuss the upper and lower bounds of the eigenvalues of the general sum-connectivity matrix. We will be needing the following lemma to prove the tight bounds. 
Lemma 3.1 [3] If $\mathrm{M}$ is a non-negative irreducible symmetric matrix with exactly two distinct eigenvalues, then there must exist a positive vector $\mathbf{u}$ and a scaler $s$, such that $\mathbf{M}=\mathbf{u u}^{T}+s \mathrm{I}$, where $\mathrm{I}$ is the identity matrix.

We first prove the tight upper bound of the largest eigenvalue $\mu_{n}$ of the general sum-connectivity matrix $\mathrm{S}_{\alpha}(G)$.

Theorem 3.1 The upper bound of the largest eigenvalue $\mu_{n}$ of the general sumconnectivity matrix $\mathrm{S}_{\alpha}(G)$ is given by

$$
\mu_{n} \leqslant \sqrt{\frac{2(n-1)}{n} \sum_{v_{i} v_{j} \in E}\left(d_{i}+d_{j}\right)^{2 \alpha}},
$$

where the equality holds if and only if $G$ is a complete graph.

Proof First, according to (2.1) and Cauchy-Schwarz inequality, we have

$$
\mu_{n}^{2}=\left(-\sum_{i=1}^{n-1} \mu_{i}\right)^{2} \leqslant(n-1) \sum_{i=1}^{n-1} \mu_{i}^{2} .
$$

The equality holds if and only if $\mu_{1}=\cdots=\mu_{n-1}$.

Second, according to (2.2), we have

$$
\sum_{i=1}^{n-1} \mu_{i}^{2} \leqslant 2 \sum_{v_{i} v_{j} \in E}\left(d_{i}+d_{j}\right)^{2 \alpha}-\mu_{n}^{2} .
$$

Therefore,

$$
\mu_{n}^{2} \leqslant(n-1)\left\{2 \sum_{v_{i} v_{j} \in E}\left(d_{i}+d_{j}\right)^{2 \alpha}-\mu_{n}^{2}\right\}
$$

It yields

$$
\mu_{n} \leqslant \sqrt{\frac{2(n-1)}{n} \sum_{v_{i} v_{j} \in E}\left(d_{i}+d_{j}\right)^{2 \alpha}}
$$

If $G$ is a complete graph $K_{n}$, then its general sum-connectivity matrix $\mathrm{S}_{\alpha}\left(K_{n}\right)=$ $(2 n-2)^{\alpha}(\mathrm{J}-\mathrm{I})$, where $\mathrm{J}$ is the all-1 matrix of order $n$. The eigenvalues of $\mathrm{S}_{\alpha}\left(K_{n}\right)$ are $\mu_{1}=\cdots=\mu_{n-1}=-(2 n-2)^{\alpha}, \mu_{n}=(n-1)(2 n-2)^{\alpha}$. Furthermore, $d_{i}+d_{j}=$ $2(n-1), \forall i \neq j$, and there are $\frac{n(n-1)}{2}$ edges in $K_{n}$. So, the equality of (3.1) holds.

If the equality of (3.1) holds, according to the above analysis and (2.1), then either $\mu_{i}=0, i=1, \cdots, n$, or $\mu_{1}=\cdots=\mu_{n-1}$ and $\mu_{n-1} \neq \mu_{n}$. The former case can be ruled out since it violates the assumption that $G$ is non-empty, and the latter case implies that $\mathrm{S}_{\alpha}(G)$ has exactly two distinct eigenvalues. In addition, $\mathrm{S}_{\alpha}(G)$ is nonnegative irreducible symmetric since $G$ is connected. According to Lemma 3.1, there 
exist an $n$-dimensional positive vector $\mathbf{u}^{T}=\left(u_{1}, \cdots, u_{n}\right)$ and a scaler $s$ such that $\mathrm{S}_{\alpha}(G)=\mathbf{u u}^{T}+s \mathrm{I}$, where $\mathrm{I}$ is the $n$-dimensional identity matrix. Since that all entries on the main diagonal of $\mathrm{S}_{\alpha}(G)$ are 0 , we obtain $s_{i i}^{\alpha}=u_{i}^{2}+s=0, i=1, \cdots, n$. Hence $u_{i}=\sqrt{-s}$, where $s<0$. Moreover,

$$
s_{i j}^{\alpha}=u_{i} u_{j}=\sqrt{-s} \sqrt{-s}=-s>0, \quad \forall i \neq j .
$$

It implies $v_{i} v_{j} \in E(G), \forall i \neq j$. Therefore $G$ is a complete graph.

We then prove the tight lower bound of the largest eigenvalue $\mu_{n}$ of the general sum-connectivity matrix $\mathrm{S}_{\alpha}(G)$.

Theorem 3.2 The lower bound of the largest eigenvalue $\mu_{n}$ of the general sumconnectivity matrix $\mathrm{S}_{\alpha}(G)$ is given by

$$
\mu_{n} \geqslant \frac{2}{n} \mathcal{Z}_{\alpha}(G)
$$

where the equality holds if and only if $G$ is a regular graph.

Proof According to Rayleigh's Principle, $\mu_{n} \geqslant \frac{\mathbf{e}^{T} \mathrm{~S}_{\alpha}(G) \mathbf{e}}{\mathbf{e}^{T} \mathbf{e}}=\frac{2 \mathcal{Z}_{\alpha}(G)}{n}$, where $\mathbf{e}$ is the all-ones vector. The equality holds if and only if $\mathbf{e}$ is an eigenvector of $\mu_{n}$, which implies that $G$ is a regular graph.

According to the upper and lower bounds of $\mu_{n}$, we establish the relationship between $\mu_{n}$ and the general product-connectivity index.

Corollary 3.1 The largest eigenvalue $\mu_{n}$ of $\mathrm{S}_{\alpha}(G)$ can be bounded by the general product-connectivity index $\mathcal{X}_{\alpha}(G)$.

- When $\alpha \leqslant 0, \mu_{n} \leqslant \sqrt{\frac{2^{2 \alpha+1}(n-1)}{n} \mathcal{X}_{\alpha}(G)}$. The equality holds if and only if $G$ is $a$ complete graph.

- When $\alpha \geqslant 0, \mu_{n} \geqslant \frac{2^{\alpha+1}}{n} \mathcal{X}_{\frac{\alpha}{2}}(G)$. The equality holds if and only if $G$ is a regular graph.

Proof First, when $\alpha \leqslant 0$,

$$
\sum_{v_{i} v_{j} \in E}\left(d_{i}+d_{j}\right)^{2 \alpha} \leqslant \sum_{v_{i} v_{j} \in E}\left(4 d_{i} d_{j}\right)^{\alpha}=4^{\alpha} \mathcal{X}_{\alpha}(G),
$$

together with (3.1), we obtain

$$
\mu_{n} \leqslant \sqrt{\frac{2(n-1)}{n} \sum_{v_{i} v_{j} \in E}\left(d_{i}+d_{j}\right)^{2 \alpha}} \leqslant \sqrt{\frac{2^{2 \alpha+1}(n-1)}{n} \mathcal{X}_{\alpha}(G)} .
$$

The equality holds if and only if $G$ is a complete graph. 
Second, when $\alpha \geqslant 0$, according to (3.2),

$$
\mu_{n} \geqslant \frac{2}{n} \mathcal{Z}_{\alpha}(G) \geqslant \frac{2}{n} \sum_{v_{i} v_{j} \in E}\left(2 \sqrt{d_{i} d_{j}}\right)^{\alpha}=\frac{2^{\alpha+1}}{n} \mathcal{X}_{\frac{\alpha}{2}}(G) .
$$

The equality holds if and only if $d_{i}=d_{j}, \forall v_{i} v_{j} \in E(G)$, which implies $G$ is a regular graph.

In the following we discuss the upper bound of spectral diameter $\mu_{n}-\mu_{1}$ of $\mathrm{S}_{\alpha}(G)$. We will be needing the following lemma.

Lemma 3.2 Let the diameter of a graph $G$ be $d$. If $\mathrm{S}_{\alpha}(G)$ has exactly $k$ distinct eigenvalues, then $k \geqslant d+1$.

Proof Let $\mu_{1}, \mu_{2}, \cdots, \mu_{k}$ be all distinct eigenvalues of $\mathrm{S}_{\alpha}(G)$, then the corresponding minimal polynomial $\varphi(\mu)$ is

$$
\varphi(\mu)=\left(\mu-\mu_{1}\right)\left(\mu-\mu_{2}\right) \cdots\left(\mu-\mu_{k}\right)=\mu^{k}+b_{1} \mu^{k-1}+\cdots+b_{k},
$$

and the following equations hold:

$$
\left(\mathrm{S}_{\alpha}(G)\right)^{k+t}+b_{1}\left(\mathrm{~S}_{\alpha}(G)\right)^{k+t-1}+\cdots+b_{k}\left(\mathrm{~S}_{\alpha}(G)\right)^{t}=0, \quad \forall t=0,1,2, \cdots
$$

where the coefficients $b_{1}, b_{2}, \cdots, b_{k}$ are real numbers.

Let us consider the entries in the $i$ th row and $j$ th column of general sumconnectivity matrix $\mathrm{S}_{\alpha}(G)$ and the adjacency matrix $\mathrm{A}(G)$. Note that $s_{i j}^{\alpha}=0$ if and only if $a_{i j}=0$. Let $\mathrm{S}_{\alpha}^{t}(G)$ and $\mathrm{A}^{t}(G)$ denote the $t$ th power of $\mathrm{S}_{\alpha}(G)$ and $\mathrm{A}(G)$, respectively. We denote the entries in the $i$ th row and $j$ th column of $\mathrm{S}_{\alpha}^{t}(G)$ and $\mathrm{A}^{t}(G)$ by $\left(s_{i j}^{\alpha}\right)^{(t)}$ and $a_{i j}^{(t)}$, respectively. Note that $a_{i j}^{(t)}$ is equal to the number of walks of length $t$ connecting vertices $v_{i}$ and $v_{j}$ in graph $G$, and $\left(s_{i j}^{\alpha}\right)^{(t)}$ counts the same walks but with different weight. Therefore, $\left(s_{i j}^{\alpha}\right)^{(t)}=0$ if and only if $a_{i j}^{(t)}=0$, $\forall t=0,1,2, \cdots$.

For contradiction, let us assume there exists graph $G$ such that $k \leqslant d$. Since the diameter of $G$ is $d$, there must exist vertices $v_{i}$ and $v_{j}$, such that $a_{i j}^{(t)}=0, \forall t<d$ and $a_{i j}^{(d)} \neq 0$. So, $\left(s_{i j}^{\alpha}\right)^{(t)}=0, \forall t<d$ and $\left(s_{i j}^{\alpha}\right)^{(d)} \neq 0$.

Now let's have $t=d-k \geqslant 0$ plug into (3.3). Since $\left(s_{i j}^{\alpha}\right)^{(d-1)}=\cdots=\left(s_{i j}^{\alpha}\right)^{(d-k)}=$ 0 , we conclude that $\left(s_{i j}^{\alpha}\right)^{(d)}=0$, and a contradiction occurs. Therefore, $k \geqslant d+1$.

Now we are ready to derive the upper bound of the spectra diameter $\mu_{n}-\mu_{1}$ of $\mathrm{S}_{\alpha}(G)$.

Theorem 3.3 The upper bound of the spectra diameter $\mu_{n}-\mu_{1}$ of $\mathrm{S}_{\alpha}(G)$ is given by

$$
\mu_{n}-\mu_{1} \leqslant 2 \sqrt{\sum_{v_{i} v_{j} \in E}\left(d_{i}+d_{j}\right)^{2 \alpha}},
$$

where the equality holds if and only if $G$ is a complete bipartite graph. 
Proof According to (2.2), we have

$$
\mu_{1}^{2} \leqslant 2 \sum_{v_{i} v_{j} \in E}\left(d_{i}+d_{j}\right)^{2 \alpha}-\mu_{n}^{2},
$$

where the equality holds if and only if $\mu_{2}=\cdots=\mu_{n-1}=0$. Note that according to $(2.1), \mu_{2}=\cdots=\mu_{n-1}=0$ implies $\mu_{1}<0, \mu_{n}>0$ and $\mu_{1}=-\mu_{n}$. Therefore,

$$
\mu_{1} \geqslant-\sqrt{2 \sum_{v_{i} v_{j} \in E}\left(d_{i}+d_{j}\right)^{2 \alpha}-\mu_{n}^{2}}
$$

and

$$
\mu_{n}-\mu_{1} \leqslant \mu_{n}+\sqrt{2 \sum_{v_{i} v_{j} \in E}\left(d_{i}+d_{j}\right)^{2 \alpha}-\mu_{n}^{2}} .
$$

According to the Cauchy-Schwarz inequality,

$$
\mu_{n}+\sqrt{2 \sum_{v_{i} v_{j} \in E}\left(d_{i}+d_{j}\right)^{2 \alpha}-\mu_{n}^{2}} \leqslant 2 \sqrt{\sum_{v_{i} v_{j} \in E}\left(d_{i}+d_{j}\right)^{2 \alpha}},
$$

where the equality holds if and only if $\mu_{n}=\sqrt{2 \sum_{v_{i} v_{j} \in E}\left(d_{i}+d_{j}\right)^{2 \alpha}-\mu_{n}^{2}}$, i.e., $\mu_{n}=$ $\sqrt{\sum_{v_{i} v_{j} \in E}\left(d_{i}+d_{j}\right)^{2 \alpha}}$. Therefore,

$$
\mu_{n}-\mu_{1} \leqslant 2 \sqrt{\sum_{v_{i} v_{j} \in E}\left(d_{i}+d_{j}\right)^{2 \alpha}} .
$$

Note that the equality holds if and only if $\mu_{1}=-\mu_{n}$ and $\mu_{2}=\cdots=\mu_{n-1}=0$, i.e., $\mathrm{S}_{\alpha}(G)$ has exactly two non-zero eigenvalues $\mu_{n}$ and $-\mu_{n}$. Hence, $\left(\mathrm{S}_{\alpha}(G)\right)^{2}$ has exactly two distinct eigenvalues: $\mu_{n}^{2}$ with multiplicity 2 and 0 with multiplicity $n-2$.

For contradiction, let us assume that $G$ is not a bipartite graph, then $G$ has an odd cycle.

Let us denote $G^{2}$ by the graph with the same set of vertices as $G$ and an edge between two vertices if and only if there is a walk of length two between them in $G$. We claim that $G^{2}$ is connected. This is due to the fact that for any pair of vertices $v_{i}$ and $v_{j}$ in $G$, if there is a walk of even length connecting them, then they are connected in $G^{2}$, otherwise we can always transfer a walk of odd length to a walk of even length by adjusting the walk on the odd cycle in $G$. Therefore $v_{i}$ and $v_{j}$ are connected in $G^{2}$.

Note that there is an edge of $G$ connecting vertices $v_{i}$ and $v_{j}$ if and only if the entry $s_{i j}^{\alpha}$ of $\mathrm{S}_{\alpha}(G)$ satisfies $s_{i j}^{\alpha} \neq 0$, and $\mathrm{S}_{\alpha}(G)$ is irreducible since $G$ is connected. Let $\mathrm{S}_{\alpha}^{2}(G)$ be the square of $\mathrm{S}_{\alpha}(G)$. So $\mathrm{S}_{\alpha}^{2}(G)$ is associated with graph $G^{2}$ and it is irreducible since $G^{2}$ is connected. According to Lemma 3.1, there must exist a positive vector $\mathbf{u}$ and a scaler $r$ such that $\mathrm{S}_{\alpha}^{2}(G)=\mathbf{u \mathbf { u } ^ { T }}+r \mathrm{I}$, where $\mathrm{I}$ is the identity matrix. Since $S_{\alpha}^{2}(G)$ is real symmetric matrix, there must exist an orthogonal matrix $\mathrm{P}$, such that $\mathrm{P}^{T}\left(\mathbf{u u}^{T}+r \mathrm{I}\right) \mathrm{P}=\operatorname{diag}\left(\mu_{n}^{2}, 0, \cdots, 0, \mu_{n}^{2}\right)$. Let $\mathbf{y}=\left(y_{1}, y_{2}, \cdots, y_{n}\right)^{T}=$ 
$\mathrm{P}^{T} \mathbf{u}$, then $\mathbf{y} \mathbf{y}^{T}=\operatorname{diag}\left(\mu_{n}^{2}-r,-r, \cdots,-r, \mu_{n}^{2}-r\right)$. Since the rank of $\mathbf{y y}^{T}$ is at most 1 , at most one of $\mu_{n}^{2}-r,-r, \cdots,-r, \mu_{n}^{2}-r$ is non-zero. Hence, all of them are zero for $n \geqslant 4$, which contradicts with the condition that $G$ is non-empty; otherwise, $n \leqslant 3$, and $G=K_{3}$ since $G$ has an odd cycle. However, it is easy to verify that the equality of (3.4) does not hold for $K_{3}$. Therefore, $G$ is a bipartite graph. In addition, according to Lemma 3.2, the diameter of $G$ is not larger than 2, so $G$ is a complete bipartite graph.

\section{Relationship Between General Sum-Connectivity Index and General Sum-Connectivity Laplacian Spectrum}

In this section we discuss the relationship between the general sum-connectivity index and the Laplacian spectrum of general sum-connectivity matrix.

Let $\mathrm{L}_{\alpha}(G)=\mathrm{D}_{\alpha}(G)-\mathrm{S}_{\alpha}(G)$ denote the general sum-connectivity Laplacian matrix of a graph $G$, where $\mathrm{D}_{\alpha}(G)=\operatorname{diag}\left(\sum_{j=1}^{n} \mathrm{~s}_{1 j}^{\alpha}, \cdots, \sum_{j=1}^{n} \mathrm{~s}_{n j}^{\alpha}\right)$. Without loss of generality, let's assume $\mathrm{L}_{\alpha}(G)$ has $t+1$ distinct eigenvalues and they are in increasing order, i.e., $\theta_{0}<\theta_{1}<\cdots<\theta_{t}$, and their multiplicities are $m_{0}, m_{1}, \cdots, m_{t}$, respectively. Note that $\mathrm{L}_{\alpha}(G)$ is a diagonally dominant matrix and has the same row sum, so the smallest eigenvalue of $\mathrm{L}_{\alpha}(G)$ is $\theta_{0}=0$, and the corresponding eigenvector is $\mathbf{e}=(1, \cdots, 1)$. In the following we show that the multiplicity of $\theta_{0}$ is 1 .

Theorem 4.1 The multiplicity of $\theta_{0}=0$ is 1 , i.e., $m_{0}=1$.

Proof Assume $\mathbf{u}=\left(u_{1}, \cdots, u_{n}\right)^{T}$ is the corresponding eigenvector of the eigenvalue $\theta_{0}=0$ of the general sum-connectivity Laplacian matrix $\mathrm{L}_{\alpha}(G)$. Without loss of generality, we assume $\left|u_{i}\right|=\max \left\{\left|u_{1}\right|, \cdots,\left|u_{n}\right|\right\}$. Since $\mathrm{L}_{\alpha}(G) \mathbf{u}=0 \mathbf{u}=0$, the $i$ th entry of $\mathrm{L}_{\alpha}(G) \mathbf{u}$ is

$$
\left(\sum_{j=1}^{n} \mathrm{~s}_{i j}^{\alpha}\right) u_{i}-\sum_{j=1}^{n} \mathrm{~s}_{i j}^{\alpha} u_{j}=0,
$$

which is equivalent to

$$
\left(\sum_{v_{i} v_{j} \in E} \mathrm{~s}_{i j}^{\alpha}\right) u_{i}-\sum_{v_{i} v_{j} \in E} \mathrm{~s}_{i j}^{\alpha} u_{j}=0
$$

So,

$$
\left|\left(\sum_{v_{i} v_{j} \in E} \mathrm{~s}_{i j}^{\alpha}\right) u_{i}\right|=\left|\sum_{v_{i} v_{j} \in E} \mathrm{~s}_{i j}^{\alpha} u_{j}\right| .
$$

Meanwhile, since $\mathrm{s}_{i j}^{\alpha}>0, \forall v_{i} v_{j} \in E(G)$, we have

$$
\left|\left(\sum_{v_{i} v_{j} \in E} \mathrm{~s}_{i j}^{\alpha}\right) u_{i}\right|=\sum_{v_{i} v_{j} \in E} \mathrm{~s}_{i j}^{\alpha}\left|u_{i}\right| .
$$


Hence

$$
\sum_{v_{i} v_{j} \in E} \mathrm{~s}_{i j}^{\alpha}\left|u_{i}\right|=\left|\sum_{v_{i} v_{j} \in E} \mathrm{~s}_{i j}^{\alpha} u_{j}\right| \leqslant \sum_{v_{i} v_{j} \in E}\left|\mathrm{~s}_{i j}^{\alpha} u_{j}\right|=\sum_{v_{i} v_{j} \in E(G)} \mathrm{s}_{i j}^{\alpha}\left|u_{j}\right|,
$$

and

$$
\sum_{v_{i} v_{j} \in E} \mathrm{~s}_{i j}^{\alpha}\left(\left|u_{i}\right|-\left|u_{j}\right|\right) \leqslant 0
$$

Since $\left|u_{i}\right| \geqslant\left|u_{j}\right|$, and $\mathrm{s}_{i j}^{\alpha}>0, \forall v_{i} v_{j} \in E(G)$, we have $\left|u_{i}\right|=\left|u_{j}\right|$. In addition, since $\sum_{j=1}^{n} \mathrm{~s}_{i j}^{\alpha} u_{i}-\sum_{j=1}^{n} \mathrm{~s}_{i j}^{\alpha} u_{j}=0, u_{i}$ and $u_{j}$ have the same sign. As $G$ is connected, we conclude that $u_{1}=\cdots=u_{n}$. Therefore the multiplicity of $\theta_{0}=0$ is 1 .

The following two theorems for a non-negative symmetric and irreducible matrix are based on the results derived by Fiedler [6] and Rodríguez [15], where $\mathbf{e}=(1, \cdots, 1)$. The results also hold for general sum-connectivity Laplacian matrix because the general sum-connectivity matrix $\mathrm{S}_{\alpha}(G)$ is a non-negative symmetric and irreducible matrix for any connected graph $G$.

Theorem 4.2 [6] The smallest positive eigenvalue $\theta_{1}$ of $\mathrm{L}_{\alpha}(G)$ satisfies

$$
\theta_{1}=2 n \min \left\{\frac{\sum_{v_{i} v_{j} \in E} \mathrm{~s}_{i j}^{\alpha}\left(\omega_{i}-\omega_{j}\right)^{2}}{\sum_{v_{i} \in V} \sum_{v_{j} \in V}\left(\omega_{i}-\omega_{j}\right)^{2}} \mid \omega \in \mathrm{R}^{n}, \omega \neq a \mathbf{e}, \forall a \in \mathrm{R}\right\} .
$$

Theorem 4.3 [15] The largest eigenvalue $\theta_{t}$ of $\mathrm{L}_{\alpha}(G)$ satisfies

$$
\theta_{t}=2 n \max \left\{\frac{\sum_{v_{i} v_{j} \in E} \mathrm{~s}_{i j}^{\alpha}\left(\omega_{i}-\omega_{j}\right)^{2}}{\sum_{v_{i} \in V} \sum_{v_{j} \in V}\left(\omega_{i}-\omega_{j}\right)^{2}} \mid \omega \in \mathrm{R}^{n}, \omega \neq a \mathbf{e}, \forall a \in \mathrm{R}\right\} .
$$

In the following we prove that general sum-connectivity index is determined by the spectrum of general sum-connectivity Laplacian matrix. In addition, we can bound the general sum-connectivity index of bipartite graphs by employing Theorems 4.2 and 4.3 .

Theorem 4.4 Let $G$ be a graph, and the spectrum of its general sum-connectivity Laplacian matrix is $\operatorname{spec}\left(\mathrm{L}_{\alpha}(G)\right)=\left\{\theta_{0}^{1}, \theta_{1}^{m_{1}}, \cdots, \theta_{t}^{m_{t}}\right\}$. Then,

1. The general sum-connectivity index of $G$ is $\mathcal{Z}_{\alpha}(G)=\frac{1}{2} \sum_{i=0}^{t} m_{i} \theta_{i}$.

2. The general sum-connectivity index of $G$ satisfies $\frac{n-1}{2} \theta_{1} \leqslant \mathcal{Z}_{\alpha}(G) \leqslant \frac{n-1}{2} \theta_{t}$, where the equality holds if and only if $G$ is a complete graph $K_{n}$.

3. Let $G=(X, Y ; E)$ be any bipartite graph, then $\frac{|X||Y|}{|X|+|Y|} \theta_{1} \leqslant \mathcal{Z}_{\alpha}(G) \leqslant \frac{|X||Y|}{|X|+|Y|} \theta_{t}$, where $|X|$ and $|Y|$ denote the number of vertices in component $X$ and $Y$, respectively. 
Proof 1. This is because

$$
\mathcal{Z}_{\alpha}(G)=\frac{1}{2} \sum_{i=1}^{n} \sum_{j=1}^{n} \mathrm{~s}_{i j}^{\alpha}=\frac{1}{2} \operatorname{tr}\left(\mathrm{L}_{\alpha}\right)=\frac{1}{2} \sum_{i=0}^{t} m_{i} \theta_{i}
$$

2. Since $0<\theta_{1}<\cdots<\theta_{t}$, we have $\frac{n-1}{2} \theta_{1} \leqslant \mathcal{Z}_{\alpha}(G)=\frac{1}{2} \sum_{i=0}^{t} m_{i} \theta_{i} \leqslant \frac{n-1}{2} \theta_{t}$, where the equality holds if and only if $t=1$, i.e., $L_{\alpha}(G)$ has exactly one non-zero eigenvalue. Together with $\theta_{0}=0, L_{\alpha}(G)$ has two distinct eigenvalues. Consequently $G$ is $K_{n}$.

3. Let $\omega=\left(\omega_{i}\right)_{i \in[n]}$, where

$$
\omega_{i}= \begin{cases}1, & v_{i} \in X \\ -1, & v_{i} \in Y .\end{cases}
$$

Then $\omega \neq a \mathbf{e}, \forall a \in \mathrm{R}$, and

$$
\sum_{v_{i} \in V} \sum_{v_{j} \in V}\left(\omega_{i}-\omega_{j}\right)^{2}=8|X||Y|
$$

By (4.1), Theorem 4.2 and Theorem 4.3, we have

$$
\begin{aligned}
\theta_{1} & \leqslant 2 n \frac{\sum_{v_{i} v_{j} \in E} \mathrm{~s}_{i j}^{\alpha}\left(\omega_{i}-\omega_{j}\right)^{2}}{\sum_{v_{i} \in V} \sum_{v_{j} \in V}\left(\omega_{i}-\omega_{j}\right)^{2}}=\frac{|X|+|Y|}{4|X||Y|} \sum_{v_{i} v_{j} \in E} \mathrm{~s}_{i j}^{\alpha}\left(\omega_{i}-\omega_{j}\right)^{2} \\
& =\frac{|X|+|Y|}{|X||Y|} \sum_{v_{i} v_{j} \in E} \mathrm{~s}_{i j}^{\alpha}=\frac{|X|+|Y|}{|X||Y|} \mathcal{Z}_{\alpha}(G)
\end{aligned}
$$

and

$$
\theta_{t} \geqslant 2 n \frac{\sum_{v_{i} v_{j} \in E} \mathrm{~s}_{i j}^{\alpha}\left(\omega_{i}-\omega_{j}\right)^{2}}{\sum_{v_{i} \in V} \sum_{v_{j} \in V}\left(\omega_{i}-\omega_{j}\right)^{2}}=\frac{|X|+|Y|}{|X||Y|} \mathcal{Z}_{\alpha}(G) .
$$

So, $\frac{|X||Y|}{|X|+|Y|} \theta_{1} \leqslant \mathcal{Z}_{\alpha}(G) \leqslant \frac{|X||Y|}{|X|+|Y|} \theta_{t}$.

\section{Conclusion}

In this paper we study the properties of general sum-connectivity index and the eigenvalues of the general sum-connectivity matrix. We leave over the lower bound of the spectra diameter $\mu_{n}-\mu_{1}$ of $\mathrm{S}_{\alpha}(G)$ as an open question. It is also interesting to explore other properties of connectivity index. In addition, more applications of connectivity index as well as other topological indices are awaiting to be explored.

Acknowledgements We thank the anonymous reviewers for their thorough reviews and insightful comments, which helped us to improve the paper. 


\section{References}

[1] Balister, P., Bollobás, B., Gerke, S.: The generalised Randić index of trees. J. Graph Theory 56(4), 270-286 (2007)

[2] Bollobás, B., Erdös, P.: Graphs of extremal weights. Ars Comb. 50, 225-233 (1998)

[3] Cao, D., Chvàtal, V., Hoffman, A.J., Vince, A.: Variations on a theorem of Ryser. Linear Algebra Appl. 260, 215-222 (1997)

[4] Delorme, C., Favaron, O., Rautenbach, D.: On the Randić index. Discrete Math. 257, 29-38 (2002)

[5] Estrada, E., Fox, M., Higham, D.J., Oppo, G.: Network Science: Complexity in Nature and Technology. Springer, London. ISBN:978-1-84996-395-4

[6] Fiedler, M.: A property of eigenvectors of nonegative symmetric matrices and its application to graph theory. Czechoslov. Math. J. 67(100), 619-633 (1975)

[7] Ghimire, J., Mani, M., Sanguankotchakorn, T., Crespi, N.: Self-connectivity estimation for super node overlay creation in ad-hoc networks. In: 2010 IEEE 17th International Conference on Telecommunications (ICT), Doha, pp. 722-727 (2010)

[8] González-Díaz, H., González-Díaz, Y., Santana, L., Ubeira, F.M., Uriarte, E.: Proteomics, networks and connectivity indices. Proteomics 8(4), 750-778 (2008). ACM Press

[9] Gutman, I., Furtula, B. (eds.) Recent Results in the Theory of Randić Index. Mathematical Chemistry Monograph, vol. 6 (2008). Kragujevac

[10] Li, X., Gutman, I.: Mathematical Aspects of Randić-Type Molecular Structure Descriptors. Mathematical Chemistry Monographs, vol. 1 (2006). Kragujevac

[11] Rajan, M.A., Chandra, M.G., Reddy, L.C., Hiremath, P.: A study of connectivity index of graph relevant to ad hoc networks. Int. J. Comput. Sci. Netw. Secur. 7(11), 198-203 (2007)

[12] Randić, M.: Characterization of molecular branching. J. Am. Chem. Soc. 97(23), 6609-6615 (1975)

[13] Ranjan, G., Zhang, Z.: Geometry of complex networks and structural centrality (2011). CoRR arXiv:1107.0989

[14] Riera-Fernández, P., Munteanu, C.R., Escobar, M., Prado-Prado, F., Martín-Romalde, R., Pereira, D., Villalba, K., Duardo-Sánchez, A., González-Díaz, H.: New Markov-Shannon entropy models to assess connectivity quality in complex networks: from molecular to cellular pathway, parasite-host, neural, industry, and legal-social networks. J. Theor. Biol. 293, 174-188 (2012)

[15] Rodríguez, J.A.: A spectral approach to the Randic index. Linear Algebra Appl. 400, 339-344 (2005)

[16] Zhou, B., Trinajstić, N.: On a novel connectivity index. J. Math. Chem. 46, 1252-1270 (2009)

[17] Zhou, B., Trinajstić, N.: On sum-connectivity matrix and sum-connectivity energy of graphs. Acta Chim. Slov. 57, 518-523 (2010) 